\title{
Reduced Capacity and Affinity of Skeletal Muscle for Insulin-mediated Glucose Uptake in Noninsulin-dependent Diabetic Subjects
}

\author{
Effects of Insulin Therapy
}

Alain D. Baron, Markku Laakso, Ginger Brechtel, and Steven V. Edelman

Departments of Medicine, Indiana University School of Medicine, Indianapolis, Indiana 46202-5124; and University of California, San Diego, San Diego, California 92093; and Veterans Administration Research Services,

Indianapolis, Indiana 46202 and San Diego, California 92161

\begin{abstract}
We have estimated the capacity and affinity of insulinmediated glucose uptake (IMGU) in whole body and in leg muscle of obese non-insulin-dependent diabetics (NIDDM, $n$ $=6$ ) with severe hyperglycemia, glycohemoglobin (GHb 14.4 $\pm 1.2 \%$ ), lean controls ( $\mathrm{ln}, n=7$ ) and obese nondiabetic controls (ob, $n=7)$. Mean \pm SEM weight $(\mathrm{kg})$ was $67 \pm 2(\mathrm{ln})$, $100 \pm 7$ (ob), and 114 \pm 11 (NIDDM), $P=$ NS between obese groups. NIDDM were also studied after 3 wk of intensive insulin therapy, GHb post therapy was $10.1 \pm 0.9, P<0.01$ vs. pretherapy. Insulin $\left(120 \mathrm{mu} / \mathrm{m}^{2}\right.$ per $\left.\mathrm{min}\right)$ was infused and the arterial blood glucose $(G)$ sequentially maintained at $\sim 4,7$, 12, and $21 \mathrm{mmol} /$ liter utilizing the $G$ clamp technique. Leg glucose uptake (LGU) was calculated as the product of the femoral arteriovenous glucose difference (FAVGd) and leg blood flow measured by thermodilution. Compared to $\mathrm{ln}, \mathrm{ob}$ and NIDDM had significantly lower rates of whole body IMGU and LGU at all G levels. Compared to ob, the NIDDM exhibited $\sim 50 \%$ and $\sim \mathbf{4 0 \%}$ lower rates of whole body IMGU over the first two $G$ levels $(P<0.02)$ but did not differ at the highest G, $P=$ NS. LGU was $83 \%$ lower in NIDDM vs. ob, $P<0.05$ at the first $G$ level only. After insulin therapy NIDDM were indistinguishable from ob with respect to whole body IMGU or LGU at all $G$ levels. A significant correlation was noted between the percent $\mathbf{G H b}$ and the $\mathbf{E G}_{\mathbf{5 0}}$ (G at which $1 / 2$ maximal FAVGd occurs) $r=0.73, P<0.05$. Thus, $(a)$ insulin resistance in NIDDM and obese subjects are characterized by similar decreases in capacity for skeletal muscle IMGU, but differs in that poorly controlled NIDDM display a decrease in affinity for skeletal muscle IMGU, and (b) this affinity defect is related to the degree of antecedent glycemic control and is reversible with insulin therapy, suggesting that it is an acquired defect. ( $J$. Clin. Invest. 1991. 87:1186-1194.) Key words: blood flow • glucose kinetics $\bullet$ insulin resistance $\bullet$ obesity
\end{abstract}

Address correspondence to Alain D. Baron, Indiana University School of Medicine, Dept. of Medicine, Division of Endocrinology and Metabolism, Emerson Hall Room 421, 545 Barnhill Dr., Indianapolis, IN 46202-5124.

Received for publication 16 April 1990 and in revised form 3 October 1990

J. Clin. Invest.

(C) The American Society for Clinical Investigation, Inc.

0021-9738/91/04/1186/09 \$2.00

Volume 87, April 1991, 1186-1194

\section{Introduction}

Non-insulin-dependent diabetes mellitus (NIDDM) ${ }^{1}$ is characterized by decreased rates of in vivo insulin-mediated glucose uptake (IMGU). Whereas the mechanism(s) responsible for this reduction in insulin action is still largely unknown, there is good agreement that the locus for this defect resides chiefly in skeletal muscle (1-4). In addition, although reduced cellular insulin receptors have been documented in isolated adipocytes from NIDDM subjects, it is widely accepted that the major defect in insulin action resides at a postbinding site (5). Much work has focused on the glucose transport system as a potential rate-limiting step in insulin action. Indeed, glucose transport was found to be reduced in both isolated adipocytes $(6,7)$ and skeletal muscle (3) from NIDDM subjects. Recently, Garvey et al. (6) demonstrated that this decrease in glucose transport was the result of a reduction in both number and functional activity of glucose transporters in adipocytes obtained from these subjects. Given that glucose transport follows saturation kinetics, these data would predict a decrease in transport capacity $\left(V_{\max }\right)$ but they cannot exclude a decreased affinity $\left(K_{\mathrm{m}}\right)$ of the glucose transport system for glucose (8).

There are currently no in vitro or in vivo data exploring the kinetic characteristics of IMGU in NIDDM. We have recently shown that in vivo skeletal muscle IMGU follows saturation kinetics with a $K_{\mathrm{m}}$ of $\sim 5-6 \mathrm{mM}$ characteristic for cellular glucose transport (9), and that obesity is associated with a reduction in $V_{\max }$ but a normal $K_{\mathrm{m}}(10)$. Given our ability to measure the kinetics of in vivo glucose uptake, the current study was undertaken to examine the kinetic parameters of IMGU in NIDDM subjects. To this end, rates of whole body and leg skeletal muscle IMGU were measured over a wide range of glucose concentrations utilizing the glucose clamp technique during a constant insulin infusion in nondiabetic obese, and obesity-matched NIDDM subjects.

Because insulin therapy has been shown to ameliorate peripheral insulin action in these subjects $(11,12)$, these kinetic studies were repeated in a subset of NIDDM subjects after a period of intensive insulin therapy.

\section{Methods}

Materials. Porcine monocomponent insulin was supplied by the Eli Lilly Co. (Indianapolis, IN). [ ${ }^{125}$ I]Insulin was purchased from New En-

1. Abbreviations used in this paper: AVGd, arteriovenous difference for blood glucose; BF, blood flow; IMGU, insulin-mediated glucose uptake; NIDDM, non-insulin-dependent diabetes mellitus; SRIF, somatostatin. 
gland Nuclear (Boston, MA). BSA (fraction V) was obtained from Armour Pharmaceutical (Chicago, IL). Guinea pig anti-insulin antibody was kindly supplied by E. Arquilla (University of California, Irvine, CA). Somatostatin (SRIF, cyclic form) was purchased from Bachem (Torrance, CA).

Subjects. The study groups consisted of 7 lean, 7 obese, and 10 obesity-matched NIDDM men. Obesity was defined by body mass in$\operatorname{dex}\left(\mathrm{BMI}\right.$, weight $\left.(\mathrm{kg}) / \mathrm{height}(\mathrm{m})^{2}>27 \mathrm{~kg} / \mathrm{m}^{2}\right)$. Data on the lean control group have been previously published (10), likewise data on six of the seven obese nondiabetic men have previously been published (10). All lean men had normal glucose tolerance, five obese nondiabetic men had normal glucose tolerance, and two had impaired glucose tolerance as defined by the National Diabetes Data Group (13). Oral glucose tolerance tests were not performed in NIDDM subjects. While hospitalized all subjects remained active to approximate their prehospital exercise level. All subjects were chemically euthyroid, and with the exception of one mildly hypertensive NIDDM male, all subjects were normotensive and no subject had a concurrent disease or was ingesting any pharmacological agents.

The clinical characteristics of the subject groups are shown in Table I. The NIDDM group was somewhat older than the lean and obese groups, $P<0.05$. Body weight and body mass index were comparable in both obese groups. NIDDM subjects were arbitrarily separated based on their percent glycohemoglobin (GHb, normal range 4-8\%) into a mild group ( $\mathrm{GHb}<11 \%), n=4$, and a severe group ( $\mathrm{GHb}>11 \%), n$ $=6$. Five NIDDM subjects had not previously been treated with antidiabetic therapy, three were removed from insulin, and two from sulfonylurea therapy for at least $4 \mathrm{wk}$ before the study.

Diet. All subjects were fed a weight maintenance $(\sim 32 \mathrm{KCal} / \mathrm{kg}$ per d) diet with three divided feedings containing one-, two-, and twofifths of the total daily calories, given at 0800,1200 , and $1700 \mathrm{~h}$, respectively. The caloric distribution of the diet was $50 \%$ carbohydrate, $20 \%$ fat, and $30 \%$ protein, and all subjects were fed this diet for at least $48 \mathrm{~h}$ before any studies.

Protocol. The study protocol is similar to that previously published (10). In brief, each subject underwent a study designed to measure insulin-mediated whole body and leg glucose uptake (femoral arterial and venous catheterization technique) at euglycemia $(\sim 4.5 \mathrm{mmol} /$ liter) and over a wide range of plasma glucose concentrations $(\sim 8,14$, and $24 \mathrm{mmol} /$ liter). To accomplish this, insulin was infused at a rate of $120 \mathrm{mU} / \mathrm{m}^{2}$ per min starting at 7:00 AM through a catheter inserted in an antecubital vein and the serum glucose was clamped at euglycemia utilizing the glucose clamp technique (14). Throughout the study $\mathrm{K}_{2} \mathrm{HPO}_{4}$ was infused to prevent hypokalemia and hypophosphatemia. At $\sim 8: 00 \mathrm{AM}$ catheters were inserted in the right femoral artery and vein as previously described (2) to measure leg blood flow and for blood sampling. The biological effect of a $120 \mathrm{mU} / \mathrm{M}^{2}$ per min insulin infusion to achieve steady-state glucose disposal occurs with a delay in controls (15) and with an even greater delay in insulin-resistant obese groups $(15,16)$, therefore, the euglycemic clamp was maintained for a sufficient period to establish steady-state conditions ( $\sim 160 \mathrm{~min})$. Throughout all studies, SRIF was infused to suppress endogenous insulin secretion as previously described (10). Rates of whole body glucose uptake were calculated during steady-state conditions from data obtained over 40-min intervals at each glucose plateau. During the combination of hyperinsulinemia- and somatostatin-induced glucagon deficiency, hepatic glucose output was assumed to be completely suppressed (5) and whole body glucose uptake was calculated from the exogenous glucose infusion rate at steady state. At hyperglycemic plateaus, rates of glucose uptake were corrected for urinary glucose loss. At each glycemic plateau, arterial and venous blood was drawn every 5 min for serum glucose determinations to obtain the mean arteriovenous glucose difference. Samples for insulin and hematocrit were drawn at the end of each plateau.

Leg glucose balance technique. Leg blood flow was measured by the thermodilution technique as previously described (2). Leg glucose uptake was calculated by applying the Fick principle (17) as the product of the femoral arteriovenous difference for blood glucose (AVGd) and the leg blood flow. The mean blood flow rates at each glycemic plateau was the average of 10 separate measurements. Because no accurate method to estimate leg muscle mass was available, we did not normalize rates of leg glucose uptake. Because obesity is associated with higher lean body mass, this has the net effect to underestimate differences between lean and obese groups. Blood glucose $(G)$ concentrations were calculated utilizing the following formula: blood $G=$ plasma $G \times[1-(0.3$ $\times \mathrm{Hct})](10)$. For technical reasons, it was not possible to obtain complete leg balance data in all subjects, therefore, leg IMGU is reported for only four subjects before and after insulin therapy. Whole body rates of IMGU are reported for six NIDDMs and AVGd data in five NIDDMs before and after insulin therapy.

Intensive insulin therapy. In six severe NIDDM subjects, the kinetics of skeletal muscle IMGU were measured after a 3-wk period of intensive insulin therapy. In five subjects, therapy consisted of twice daily injections of intermediate acting insulin and injections of regular insulin before meals. One subject was treated with continuous subcutaneous insulin infusion delivered via a portable pump. In all subjects, blood glucose concentrations were monitored seven times daily, 30 min before and $2 \mathrm{~h}$ after meals and before bedtime. Insulin dose was adjusted to achieve near normal daily glucose profiles. Insulin therapy was discontinued for $2 \mathrm{~d}$ before repeating the in vivo studies.

Table I. Characteristics of the Study Groups

\begin{tabular}{|c|c|c|c|c|c|c|}
\hline & Lean & Obese & NIDDM & $\begin{array}{c}\text { NIDDM } \\
\text { (mild) }\end{array}$ & $\begin{array}{c}\text { NIDDM } \\
\text { (severe) } \\
\text { preinsulin } \\
\text { therapy }\end{array}$ & $\begin{array}{c}\text { NIDDM } \\
\text { (severe) } \\
\text { postinsulin } \\
\text { therapy }\end{array}$ \\
\hline No. subjects & 7 & 7 & 10 & 4 & 6 & 6 \\
\hline Age $(y r)$ & $36 \pm 2$ & $39 \pm 2$ & $44 \pm 1^{*}$ & $45 \pm 2^{*}$ & $44 \pm 1^{*}$ & \\
\hline Body wt $(k g)$ & $67 \pm 2^{\ddagger}$ & $100 \pm 7$ & $109 \pm 8$ & $101 \pm 10$ & $114 \pm 11$ & $118 \pm 11$ \\
\hline Body mass index $\left(\mathrm{kg} / \mathrm{m}^{2}\right)$ & $22 \pm 1$ & $33 \pm 2$ & $36 \pm 2$ & $33 \pm 3$ & $38 \pm 3$ & $39 \pm 3$ \\
\hline \multicolumn{7}{|l|}{ Fasting serum glucose } \\
\hline concentrations (mmol/liter) & $5.01 \pm 0.1$ & $5.3 \pm 0.1$ & $13 \pm 1^{*}$ & $10.8 \pm 0.8^{*}$ & $14.3 \pm 1.7^{*}$ & $6.9 \pm 0.6^{8}$ \\
\hline \multicolumn{7}{|l|}{ Fasting insulin concentrations } \\
\hline (pmol/liter) & $36 \pm 7$ & $131 \pm 23$ & $244 \pm 67$ & $294 \pm 100$ & $158 \pm 29$ & $273 \pm 79$ \\
\hline Percent glycohemoglobin & & & $12 \pm 1.2$ & $8.5 \pm 0.5$ & $14.4 \pm 1.2^{\prime \prime}$ & $10.1 \pm 0.9^{\S}$ \\
\hline
\end{tabular}

Data are expressed as mean \pm SEM. ${ }^{*} P<0.05$ NIDDM vs. obese and lean. ${ }^{\ddagger} P<0.01$ lean vs. obese groups. ${ }^{\S} P<0.01$ NIDDM postinsulin therapy vs. preinsulin therapy. " $P<0.01$ severe vs. mild NIDDM. 
Analytical methods. Blood for serum determinations was drawn, placed in untreated polypropylene tubes, and centrifuged using a Eppendorf microcentrifuge (Brinkman Instruments, Westbury, NY). The glucose concentration of the supernatant was then measured by the glucose oxidase methods using a glucose analyzer (Yellow Springs Instruments, model 23A, Yellow Springs, $\mathrm{OH}$ ). Blood for determination of serum insulin concentration was collected in untreated tubes and allowed to clot. The specimens were spun, and the supernatant was removed and stored at $-20^{\circ}$. Serum insulin levels were measured by double antibody RIA (18). Percent glycosylated hemoglobin (GHb) was determined using the glycaffin kit (Isolab, Akron, $\mathrm{OH}$ ).

Data analysis. All calculations and analyses were performed using the CLINFO data base management and analysis program (Bolt Beranek, and Newman, Inc., Cambridge, MA) operational at the University of California, San Diego General Clinical Research Center and SPSS/PC+ programs (SPSS Inc., Chicago, IL). The data are presented as mean \pm SEM. Overall analysis of variance was performed by the Kruskal-Wallis test, the comparison between two groups was performed with the Mann-Whitney test. Pre- and posttreatment data were compared by the Wilcoxon matched pairs sign-rank test. The curves for whole body and leg glucose uptake, and AVGd vs. glucose concentration were fitted to a four-parameter logistic equation using a least mean square iterative routine (ALLFIT) (19): $Y=\left(V_{\max }-D\right) /(1$ $=\left(V_{\max }-D\right) /\left(1+\left(G / K_{\mathrm{m}}\right)^{B}\right)+D$, where $V_{\max }=$ expected maximal response, $D=$ expected minimal response, $G=$ glucose concentration, $K_{\mathrm{m}}=$ glucose concentration with expected response halfway between $V_{\max }$ and $D, B=$ slope factor, and $Y=$ the rate of whole body or leg glucose uptake, or AVGd across the leg. The only assumption in the analysis was that $Y=0$ for whole body, leg glucose uptake and for AVGd at 0 glucose concentration. The runs test was calculated on the basis of the individual curves both in lean and obese subjects separately to evaluate the goodness to fit. The runs test for each curve is a test of randomness of the residuals. In all subject groups, the data points were randomly distributed around the fitted curve $(P$ value for the runs test was $>0.05$ ). The best fit for whole body and leg glucose uptake, and AVGd vs. glucose concentration was determined on the basis of individual data.

\section{Results}

Glucose and Insulin Concentrations During the Glucose Clamp Studies (Table II)

The desired arterial glucose concentrations for each glycemic plateau were successfully achieved with a coefficient variation of $<4 \%$ in all cases. Although serum insulin concentrations tended to be somewhat higher in the obese groups, there were no statistical differences between subject groups in serum insulin and blood glucose concentrations.

\section{Rates of Whole Body Glucose Uptake (Fig. 1)}

Compared to lean controls, rates of whole body IMGU were reduced at all glycemic plateaus in both obese nondiabetic and in obese diabetic groups before and after insulin therapy. Compared to obese controls, rates of whole body IMGU in the NIDDM group as a whole were lower by $54 \%(P<0.001)$ at the first, $41 \%(P<0.01)$ at the second, and 33\% $(P<0.05)$ at the third glycemic plateaus. However, no difference was observed in the rates of whole body IMGU at the fourth and highest glycemic levels between obese nondiabetic and diabetic groups, $P=$ NS. Compared to obese nondiabetics, rates of whole body IMGU in mild NIDDM were reduced by $43 \%(P<0.005)$ and $31 \%(P<0.05)$ at the first and second glycemic plateaus, respectively, but were not different at the two highest glycemic levels, $P=$ NS. Rates of whole body IMGU in severe NIDDMs vs. obese nondiabetics (Fig. 1) were reduced by $62 \%$ at the first $(P$ $<0.001), 41 \%$ at the second $(P<0.01)$ and $38 \%$ at the third $(P$ $<0.05)$ glycemic level but were not different at the fourth and highest glycemic level, $P=$ NS. Therefore, rates of whole body IMGU at the highest glucose level were not different among obese groups irrespective of the diabetic status.

After intensive insulin therapy, rates of whole body IMGU

Table II. Arterial Blood Glucose and Serum Insulin Concentrations during Clamp Studies in Lean, Obese Nondiabetic and Obese Diabetic Groups

\begin{tabular}{|c|c|c|c|c|}
\hline & \multicolumn{4}{|c|}{ Target blood glucose concentrations (mmol/liter) } \\
\hline & 4.0 & 7.0 & 12 & 21 \\
\hline \multicolumn{5}{|l|}{ Glucose (mmol/liter) } \\
\hline Lean $(n=7)$ & $4.0 \pm 0.1$ & $7.2 \pm 0.1$ & $12.1 \pm 0.4$ & $21.1 \pm 0.5$ \\
\hline Obese $(n=7)$ & $3.9 \pm 0.1$ & $7.4 \pm 0.1$ & $12.1 \pm 0.3$ & $20.8 \pm 0.4$ \\
\hline $\operatorname{NIDDM}(n=10)$ & $3.9 \pm 0.1$ & $7.4 \pm 0.1$ & $12.4 \pm 0.1$ & $20.5 \pm 0.4$ \\
\hline NIDDM (mild, $n=4)$ & $4.0 \pm 0.1$ & $7.4 \pm 0.1$ & $12.2 \pm 0.2$ & $20.4 \pm 0.3$ \\
\hline NIDDM (severe, $n=6$ ) & $3.9 \pm 0.1$ & $7.4 \pm 0.1$ & $12.5 \pm 0.2$ & $20.5 \pm 0.7$ \\
\hline $\begin{array}{l}\text { NIDDM (severe } n=6 \\
\text { postinsulin therapy) }\end{array}$ & $3.9 \pm 0.0$ & $7.7 \pm 0.1$ & $13.1 \pm 0.1$ & $21.2 \pm 0.5$ \\
\hline$P$ value* & NS & NS & NS & NS \\
\hline \multicolumn{5}{|l|}{ Insulin (pmol/liter) } \\
\hline Lean & $1845 \pm 134$ & $2019 \pm 260$ & $1876 \pm 105$ & $2009 \pm 187$ \\
\hline Obese & $2419 \pm 197$ & $2378 \pm 125$ & $2122 \pm 180$ & $2368 \pm 167$ \\
\hline NIDDM & $2562 \pm 256$ & $2412 \pm 209$ & $2438 \pm 225$ & $2480 \pm 276$ \\
\hline NIDDM (mild) & $2673 \pm 406$ & $2313 \pm 206$ & $2457 \pm 239$ & $2583 \pm 279$ \\
\hline NIDDM (severe) & $2490 \pm 357$ & $2478 \pm 334$ & $2426 \pm 359$ & $2411 \pm 377$ \\
\hline $\begin{array}{l}\text { NIDDM (severe, } \\
\text { postinsulin therapy) }\end{array}$ & $3069 \pm 439$ & $3032 \pm 456$ & $2742 \pm 380$ & $2732 \pm 431$ \\
\hline$P$ value* & NS & NS & NS & NS \\
\hline
\end{tabular}

Data are expressed as mean \pm SEM. * Significance of the difference between lean, obese, mild NIDDM and severe NIDDM pretherapy (KruskalWallis analysis of variance). 


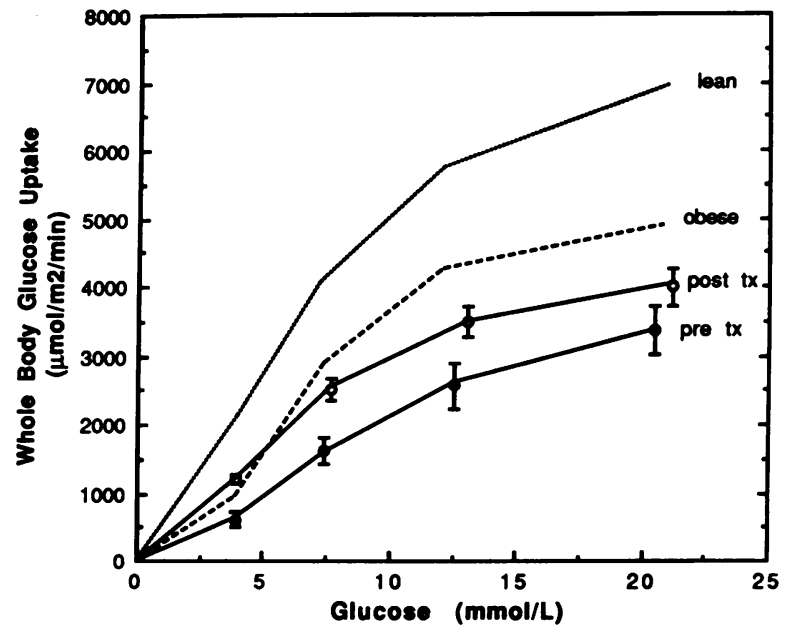

Figure 1. Rates of whole body glucose uptake during a $120 \mathrm{mu} / \mathrm{m}^{2}$ per min insulin infusion as a function of the prevailing blood glucose concentration in a group of lean controls (dotted line), obese nondiabetics (dashed line), obese non-insulin-dependent diabetics before (pre tx, solid circles) and after (post tx, open circles) $3 \mathrm{wk}$ of intensive insulin therapy. The lines represent the best fit based on a four-parameter logistic equation (ALLFIT, 19).

in the severe NIDDM group increased over pretreatment values by $97 \%(P<0.01), 50 \%(P<0.05)$ and $36 \%(P<0.05)$ at the first, second, and third glycemic plateaus, respectively. In contrast, rates of whole body IMGU at the highest glycemic plateau were unchanged after insulin treatment and not different from those observed in obese controls or mild NIDDMs.

\section{Leg Balance Data (Fig. 2, A-C)}

(a) Femoral arteriovenous glucose differences (Fig. 2 A). Compared to lean controls, the femoral AVGd was reduced in nondiabetic and diabetic obese groups at all glycemic levels. Compared with obese nondiabetics, the femoral AVGd in the NIDDM group as a whole was reduced by $59 \%(0.86 \pm 0.11$ vs. $0.35 \pm 0.13 \mathrm{mmol} /$ liter, $P<0.01$ ) at the first glycemic plateau but not different $(P=\mathrm{NS})$ at the other glycemic levels. Mild NIDDMs were indistinguishable from nondiabetic obese with respect to the femoral AVGd at all glycemic levels. In contrast, severe NIDDM had a marked reduction in femoral AVGd at the first $(83 \%, P<0.001)$ and second $(41 \%, P<0.05)$ glycemic plateau when compared with obese controls. The femoral AVGd at the third and highest glycemic plateau was similar in obese nondiabetic and severe NIDDM groups, $P=$ NS.

In the severe NIDDM group, intensive insulin therapy lead to a marked increase in the femoral AVGd obtained at the first two glycemic plateaus, to values similar to those obtained in obese controls and mild NIDDM (Fig. $2 A$ ) but had no effect on the AVGd obtained at the highest glucose level. Thus, the femoral AVGd at the highest glycemic level was similar in all obese groups irrespective of diabetes.

(b) Leg blood flow rates (Fig. 2 B). As previously reported (10), leg blood flow (BF) increased progressively as the plasma glucose concentration was raised in lean control subjects $(P$ $<0.05$ ). In contrast there was no significant trend for leg BF rates in the obese nondiabetic or diabetic groups. Compared to lean controls, leg BF in obese nondiabetics was lower only at the fourth glycemic plateau $(P<0.05)$ and in NIDDMs as a
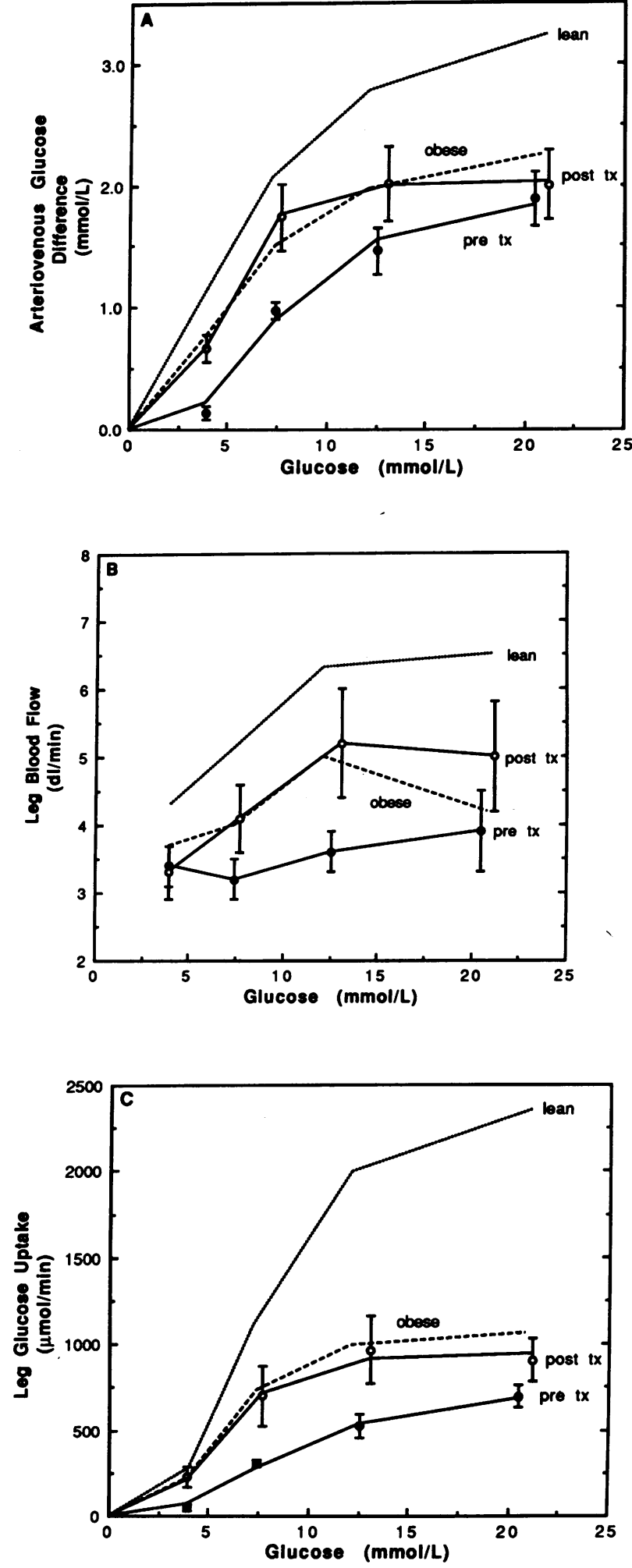

Figure 2. Femoral arteriovenous glucose difference $(A)$, leg blood flow $(B)$, and leg glucose uptake $(C)$ during a $120 \mathrm{mu} / \mathrm{m}^{2}$ per min insulin infusion as a function of the prevailing blood glucose concentration in lean controls (dotted line), obese nondiabetics (dashed line) and obese non-insulin-dependent diabetics before (pre tx, solid circles) and after (post tx, open circles) 3 wk of intensive insulin therapy. The lines represent the best fit based on a four-parameter logistic equation (ALLFIT, 19). 
whole, only at the third and fourth glycemic plateaus $(P$ $<0.05)$. At each respective glucose plateau leg BF in severe NIDDMs was lower than in lean controls by $14 \%(P<0.05)$, 24\% $(P<0.05), 33 \%(P<0.01)$, and $38 \%(P<0.01)$. Leg BF rates were not different among the obese nondiabetic and diabetic groups at any glucose level studied.

Although insulin therapy in severe NIDDM had a tendency to increase leg $B F$ rates, this improvement did not reach statistical significance and thus rates of leg BF in these NIDDMs remained significantly lower than rates of leg blood flow in lean controls.

(c) Leg glucose uptake (Fig. 2 C). Compared to lean controls, rates of leg IMGU were reduced in obese nondiabetic and diabetic groups at all glycemic plateaus. Compared with obese nondiabetics, rates of leg IMGU were not different in the NIDDM group as a whole except at the first glycemic plateau, where rates of leg IMGU were reduced by $50 \%(P<0.05)$. Rates of leg IMGU were not different between mild NIDDM and obese nondiabetics at all glycemic levels. Compared to obese nondiabetics, severe NIDDMs displayed an $84 \%$ reduction $(P<0.01)$ in rates of leg IMGU at the first glycemic plateau, but these rates were not different at other glucose levels studied, $P=$ NS.

After insulin therapy, rates of leg IMGU in severe NIDDMs improved to values indistinguishable from those observed in obese controls or mild NIDDMs (data not shown). As in the case of whole body IMGU and femoral AVGd, rates of leg IMGU at the highest glycemic plateau were not statistically different among obese groups.

\section{Kinetic Analysis}

The apparent $V_{\max }$ and $K_{\mathrm{m}}$ values, estimated by curve fitting analysis (19) are shown in Table III.

\section{Whole Body IMGU}

Compared to lean subjects $V_{\max }$ values for whole body IMGU were $\sim 35 \%$ lower in obese nondiabetics $(P<0.01), \sim 45 \%$ lower in NIDDM as a whole $(P<0.001), \sim 50 \%$ lower in mild $\operatorname{NIDDM}(P<0.001)$, and $\sim 41 \%$ lower in severe NIDDMs $(P$ $<0.001)$. Interestingly, there were no statistical differences in $V_{\max }$ values between obese control and their diabetic counterparts, although the latter tended to have somewhat lower values. Insulin therapy in severe NIDDM had no effect on the $V_{\max }$ for whole body IMGU. Compared with obese controls, $K_{\mathrm{m}}$ values for whole body IMGU were somewhat higher in the NIDDM group as a whole, although these were not statistically different, $P=$ NS. Similarly, whereas the $K_{\mathrm{m}}$ value for whole body IMGU tended to be elevated in severe NIDDM compared with the other obese groups, it did not reach statistical significance.

\section{Leg IMGU}

As with rates of whole body IMGU, $V_{\max }$ values for leg IMGU were lower in all obese groups compared with lean controls $(P$ $<0.01$ ). Similarly, there was no difference in $V_{\max }$ between obese nondiabetics and diabetic groups irrespective of insulin therapy. $K_{\mathrm{m}}$ values for leg IMGU were not different from lean controls in obese nondiabetics and in the diabetic group as a whole. However, the $K_{\mathrm{m}}$ for severe NIDDM preinsulin therapy was significantly higher than that observed in obese controls or mild NIDDMs $(P<0.05)$. After insulin therapy the $K_{\mathrm{m}}$ decreased (i.e., affinity increased) to a value similar to that seen in both lean and obese controls and mild NIDDMs.

As we have previously pointed out (9), it is important to note that kinetic parameters estimated from whole body or limb balance data do not strictly reflect the kinetic characteristics of tissue glucose uptake per se but also include a hemodynamic component, i.e., blood flow or insulin and glucose delivery to insulin-sensitive tissues. Because blood flow increased over the range of glucose plateaus in lean subjects and fluctuated in other groups, it is more accurate to estimate tissue kinetic characteristics by examining the femoral AVGd data, i.e., tissue glucose uptake independent of glucose and insulin delivery. Because the AVGd does not describe a rate or veloc-

Table III. Apparent $V_{\max }$ and $K_{m}$ Values for Whole Body and Leg Insulin-mediated Glucose Uptake and Femoral Arteriovenous Glucose Difference

\begin{tabular}{|c|c|c|c|c|c|c|c|}
\hline & $\begin{array}{l}\text { Lean } \\
n=7\end{array}$ & $\begin{array}{l}\text { Obese } \\
n=7\end{array}$ & $\begin{array}{c}\text { NIDDM } \\
n=10\end{array}$ & $\begin{array}{l}\text { Mild NIDDM } \\
\quad n=4\end{array}$ & $\begin{array}{l}\text { Severe NIDDM } \\
\text { pretherapy } \\
n=6\end{array}$ & $\begin{array}{c}\text { Severe NIDDM } \\
\text { posttherapy } \\
n=6\end{array}$ & $P$ value* \\
\hline \multicolumn{8}{|l|}{ Whole body glucose disposal } \\
\hline$V_{\max }\left(\mu \mathrm{mol} / \mathrm{m}^{2}\right.$ per min $)$ & $7902 \pm 256$ & $5125 \pm 446^{\ddagger}$ & $4346 \pm 414^{\ddagger}$ & $3928 \pm 644^{\ddagger}$ & $4625 \pm 556^{\ddagger}$ & $4499 \pm 398^{\ddagger}$ & 0.002 \\
\hline$K_{\mathrm{m}}(\mathrm{mmol} /$ liter $)$ & $7.0 \pm 0.4$ & $6.7 \pm 1.2$ & $10.3 \pm 2.2$ & $7.1 \pm 1.2$ & $12.4 \pm 3.3$ & $7.2 \pm 0.6$ & 0.157 \\
\hline Leg IMGU & & & & & $n=5$ & $n=4$ & \\
\hline$V_{\max }(\mu \mathrm{mol} / \mathrm{min}$ per leg $)$ & $2444 \pm 266$ & $1081 \pm 233^{\ddagger}$ & $818 \pm 83^{\ddagger}$ & $892 \pm 176^{\ddagger}$ & $758 \pm 67^{\ddagger}$ & $982 \pm 165^{\ddagger}$ & 0.004 \\
\hline$K_{\mathrm{m}}(\mathrm{mmol} /$ liter $)$ & $7.6 \pm 1.1$ & $5.9 \pm 0.7$ & $7.4 \pm 0.8$ & $5.2 \pm 0.5$ & $9.2 \pm 0.7^{\S}$ & $5.9 \pm 0.6^{11}$ & 0.016 \\
\hline $\begin{array}{l}\text { Femoral arteriovenous glucc } \\
\text { difference }\end{array}$ & & & & & $n=5$ & $n=5$ & \\
\hline AVGd-max (mmol/liter) & $3.57 \pm 0.1$ & $2.41 \pm 0.28^{\ddagger}$ & $2.03 \pm 0.25^{\ddagger}$ & $1.88 \pm 0.34^{\ddagger}$ & $2.14 \pm 0.38^{\ddagger}$ & $2.06 \pm 0.32^{\ddagger}$ & 0.004 \\
\hline $\mathrm{EG}_{\mathrm{s0}}(\mathrm{mmol} /$ liter $)$ & $6.0 \pm 0.8$ & $5.7 \pm 0.7$ & $7.1 \pm 0.9$ & $5.1 \pm 0.4$ & $8.9 \pm 1.0^{\S}$ & $4.8 \pm 0.4^{\| \prime}$ & 0.020 \\
\hline
\end{tabular}

Data are obtained by curve fitting analysis (ALLFIT, 19) and are expressed as mean \pm SEM. As the AVGd does not represent a rate, the kinetic parameters for AVGd are expressed as AVGd-max and $E_{50}$ for maximal glucose extraction and the glucose concentration at which $1 / 2 \mathrm{AVGd}$ max is achieved, respectively. * Significance of the difference between lean, obese, mild NIDDM, and severe NIDDM pretherapy (Kruskal-Wallis analysis of variance). ${ }^{\ddagger} P<0.001$; obese controls and NIDDM vs. lean (Mann-Whitney test). ${ }^{8} P<0.05$; NIDDM (severe) vs. obese controls and mild NIDDM (Mann-Whitney test). " $P<0.05$; NIDDM postherapy vs. NIDDM pretherapy (Wilcoxon sign-rank test). 
ity, we have chosen to designate the maximal AVGd as AVGd$\max$ (rather than $V_{\max }$ ) and likewise $\mathrm{EG}_{50}$ as the glucose concentration at which $1 / 2 \mathrm{AVGd}-\max$ is obtained (rather than $K_{\mathrm{m}}$ ).

\section{Femoral AVGd}

As with whole body and leg IMGU, compared to lean subjects AVGd-max was reduced in obese nondiabetic and diabetic groups $(P<0.001)$, respectively. There was no difference in maximal leg muscle extraction capacity (AVGd-max) between the obese nondiabetic and diabetic groups. In the severe NIDDM group insulin therapy had no effect to modulate AVGd-max.

The $\mathrm{EG}_{50}$ values for lean and obese controls and the NIDDM group as a whole were not statistically different, ranging between $5.7 \pm 0.7$ and $7.1 \pm 0.9 \mathrm{mmol} / \mathrm{liter}$. In contrast, the $E_{50}$ for severe NIDDMs before insulin treatment was nearly twice as high $(8.9 \pm 1.0 \mathrm{mmol} / \mathrm{liter}, P<0.05)$ than $\mathrm{EG}_{50}$ values in lean, obese, and mild NIDDMs. Insulin therapy lead to a normalization of the $\mathrm{EG}_{50}$ to a value $(4.8 \pm 0.4 \mathrm{mmol} / \mathrm{liter})$ similar than for lean and obese controls and mild NIDDMs but different from the pretreatment value, $P<0.05$.

It is important to note that because leg blood flow in the obese groups showed no significant trends over the range of glycemic levels, kinetic analysis of leg glucose uptake (AVGd $\times$ blood flow) gave rise to similar results as the kinetic analysis for femoral AVGd. In contradistinction, because lean controls exhibited significant increases in leg blood flow over the range of glycemic plateaus, the $K_{\mathrm{m}}$ for leg IMGU in this group was similar than the $K_{\mathrm{m}}$ for leg IMGU or $\mathrm{EG}_{50}$ for femoral AVGd in severe NIDDMs.

Relationship between Percent Glycohemoglobin (GHb) and Affinity of Tissues for Glucose Uptake (Table IV)

Because there appeared to be a general relationship between the degree of metabolic control as gauged by the percent $\mathrm{HGb}$ and the $K_{\mathrm{m}}$ or $\mathrm{EG}_{50}$ among groups, we examined the association more closely by correlating individual values of percent $\mathrm{GHb}$ with parameters of affinity for whole body and leg IMGU and femoral AVGd. As can be seen in Table IV, a significant correlation was found between the degree of antecedent glycemic control $(\% \mathrm{GHb})$ and the affinity for glucose of the glucose uptake system of insulin-sensitive tissues in the leg (i.e., skeletal muscle) as estimated by the femoral $\mathrm{AVGd} \mathrm{EG}_{50}$ in a group of nine untreated NIDDMs (AVGd data not obtained in one subject). To better illustrate this relationship, the individual data

Table IV. Correlation Analysis between Percent Glycohemoglobin $(G H b)$ and Affinity Constants $\left(K_{m}\right.$ and $\left.E G_{50}\right)$ for Whole Body and Leg IMGU and Femoral Arteriovenous Glucose Difference $(A V G d)$ in Severe NIDDM Subjects Before and After Intensive Insulin Therapy

\begin{tabular}{llc}
\hline & \multicolumn{2}{c}{ \%GHb } \\
\cline { 2 - 3 } & Before insulin therapy & After insulin therapy $(n)$ \\
\hline $\mathrm{K}_{\mathrm{m}}$, whole body IMGU & $r=0.37(10)$ & $r=0.34(6)$ \\
$K_{\mathrm{m}}$, leg IMGU & $r=0.59(10)$ & $r=0.85(4)$ \\
EG $_{\text {s0 }}$, AVGd & $r=0.72^{*}(10)$ & $r=0.90^{*}(5)$ \\
\hline
\end{tabular}

${ }^{*} P<0.05$. for femoral $\mathrm{AVGd} \mathrm{EG}_{\mathbf{s 0}}$ before and after insulin therapy are displayed in Fig. 3.

\section{Discussion}

The results of these studies indicate that reduced rates of in vivo IMGU in NIDDM subjects with poor metabolic control are largely due to reduced capacity and affinity of skeletal muscle for IMGU. Moreover, the data suggest that in kinetic terms, insulin resistance in obese NIDDM with poor glycemic control differs from that found in obese nondiabetics only by virtue of the affinity defect because the maximal capacity for skeletal muscle IMGU was similar in both obese nondiabetic and diabetic groups. Finally, a strong association was found between the degree of antecedent glycemic control (\% GHb) and the magnitude of the affinity defect for skeletal muscle IMGU and this defect was reversible with insulin therapy.

Utilizing the euglycemic hyperinsulinemic clamp technique, numerous investigators $(5,11,20-24)$ have documented reduced rates of whole body IMGU in NIDDM subjects. In their now classic studies, Kolterman et al. (5) constructed the insulin dose response curves for insulin's effect to stimulate whole body IMGU in NIDDMs and demonstrated both decreases in insulin sensitivity and responsiveness in these subjects. However, these previous studies performed at a single glucose concentration cannot define the kinetic mechanisms responsible for reduced rates of IMGU observed in these subjects. The current data indicate that obesity, regardless of the diabetic state is associated with a $\sim 40 \%$ reduction in maximal rates of whole body IMGU and $\sim 60 \%$ reduction in maximal rates of leg muscle IMGU. We have previously shown that the femoral AVGd is a more accurate reflection of the efficiency of tissue glucose uptake per se than whole body or limb glucose uptake $(9,10)$ because these latter measurements include a hemodynamic variable (blood flow) which can confound analy-

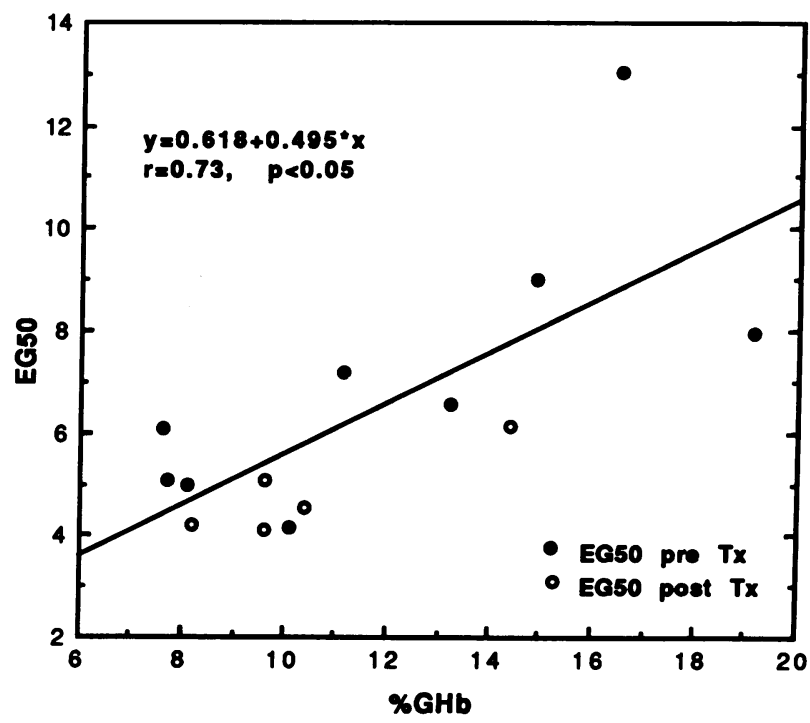

Figure 3. Correlation analysis between percent glycohemoglobin and the $\mathrm{EG}_{\mathbf{s 0}}$ (glucose concentration at which one-half the maximal femoral arteriovenous glucose difference is achieved) in a group of poorly controlled non-insulin-dependent diabetics before (solid circles) and after intensive insulin therapy (open circles). 
sis of the kinetic properties of skeletal muscle tissue per se. Moreover, we have previously validated this approach by demonstrating that increments in leg blood flow rates do not affect the femoral AVGd because under these conditions the transit time for glucose (or blood velocity) is not altered (10). Compared to lean controls, maximal femoral AVGd was decreased by $\sim 40 \%$ in obese nondiabetic and diabetic groups. However, maximal femoral AVGd was not different among obese groups. Therefore, maximal insulin-mediated skeletal muscle glucose extraction was markedly decreased in obese subjects and was not worsened by the diabetic state.

Although the rate-limiting step for in vivo IMGU in health and disease is still controversial, much evidence has recently accumulated $(9,25,26)$, suggesting that this step resides at the level of the cellular glucose transport system. In keeping with this, our data indicating a decrease in $V_{\max }$ for glucose uptake are compatible with those of Garvey et al. (6) and Karnieli et al. (27) demonstrating reduced number of glucose transporters in isolated adipocytes from obese diabetic and nondiabetic subjects but do not exclude other possibilities (e.g., glycogen synthase activity).

Perhaps the most intriguing finding of this study is that of decreased affinity of skeletal muscle for IMGU in poorly controlled NIDDMs. Indeed, in this group the $K_{\mathrm{m}}$ for leg IMGU was significantly higher and the $\mathrm{EG}_{50}$ for femoral AVGd was nearly twice that of lean and obese controls and mild NIDDM subjects. Our findings are compatible with those of Revers et al. (8), who reported that over a range of serum glucose concentrations, severely insulin-resistant NIDDMs exhibited a lesser fall in whole body insulin-mediated glucose clearance than more insulin-sensitive NIDDMs. Because glucose clearance falls when the blood glucose concentration exceeds the $K_{\mathrm{m}}$ for glucose uptake, these data would also suggest that NIDDMs have a higher $K_{\mathrm{m}}$ for IMGU than controls.

Based on the assumption that glucose transport is limiting for in vivo IMGU, our finding of decreased skeletal muscle affinity for IMGU is, at least in part, compatible with the findings of Garvey et al. (6) demonstrating a decrease in functional activity of the glucose transporter from adipocytes obtained from NIDDM subjects. Regardless of the molecular or cellular basis for our findings, it is apparent that in kinetic terms resistance to insulin's action to stimulate glucose uptake in obese nondiabetics differs from that in NIDDMs merely by virtue of this affinity defect. This novel observation is all the more robust because it is apparent whether one considers the femoral AVGd, whole body, or limb rates of IMGU.

If well controlled obese patients with NIDDM and obese nondiabetic subjects are indistinguishable with respect to their kinetic characteristics for IMGU, is it reasonable to ask what causes hyperglycemia in NIDDMs? Although the etiology of "garden variety" NIDDM is unknown, it is now well recognized that abnormalities in peripheral insulin action, insulin secretion, and accelerated rates of hepatic glucose output are required for the full expression of the NIDDM syndrome (28). Therefore, differences in kinetic parameters alone for IMGU between obese NIDDM and obese nondiabetics do not need to exist and are not sufficient for the appearance of hyperglycemia.

It is important to point out the apparent discordances between rates of whole body and leg glucose uptake. For example, compared to obese nondiabetics, rates of whole body IMGU in the NIDDM group are significantly lower at the first three glycemic plateaus, but leg glucose uptake in NIDDMs is only significantly decreased at the first glucose level. The reason for the lack of concordance between whole body and leg glucose uptake data is likely to be multifactorial: (a) Over the range of glucose concentrations studied, rates of whole body IMGU, as defined herein, represent a composite of rates of glucose uptake in insulin sensitive and non-insulin sensitive tissues, which likely exhibit a wide range of kinetic characteristics. In contrast, leg IMGU occurs predominantly in a single tissue, i.e., skeletal muscle. (b) Rates of whole body IMGU were calculated from the glucose infusion rates, whereas rates of leg IMGU are the product of the femoral AVGd and leg blood flow. Estimates of rates of leg IMGU are likely to be associated with a larger error (greater variation) than rates of whole body IMGU. (c) Rates of whole body IMGU were normalized to body surface area whereas rates of leg IMGU were not normalized. $(d)$ Due to the relatively small number of subjects studied, it is possible that type II statistical errors were committed (i.e., finding no difference when a difference exists).

Intensive insulin therapy was associated with normalization of leg tissues affinity for glucose $\left(\mathrm{EG}_{50}\right)$ but had no effect on the maximal femoral AVGd in poorly controlled NIDDMs. Thus, our data are consistent with that of previous workers (11, $12,29)$ who have reported increased rates of whole body IMGU in NIDDM subjects after intensive insulin therapy when these rates were measured at euglycemia with the glucose clamp technique. Utilizing the same technique, Garvey et al. (11) reported that insulin responsiveness (i.e., rates of whole body IMGU at maximally effective insulin concentrations) in NIDDM was ameliorated but not normalized by intensive insulin therapy. Our data, are consistent with the idea that the improvement in insulin action after insulin therapy previously reported reflects a reversal of the affinity defect and that the lack of normalization in insulin responsiveness after insulin therapy in these subjects reflects the constancy of the capacity defect.

The significant correlation between the percent $\mathrm{GHb}$ and the magnitude of the affinity defect in NIDDMs suggests that the affinity defect observed in poorly controlled NIDDMs is acquired and that it is the result directly or indirectly of poor glycemic control. Moreover, although for the purpose of discussion the NIDDM group was separated into two arbitrary groups (mild and severe) the relationship between percent $\mathrm{GHb}$ and the affinity defect describes, in fact, a single group with a spectrum of affinity defects.

In their elegant studies, Rossetti et al. (30) have demonstrated that elevated glucose concentrations per se were toxic to peripheral tissues as reflected by decreased rates of IMGU and that this defect was entirely reversible with glycemic control, independent of changes in insulin concentrations. Thus, it is possible that the affinity defect described here is the expression of glucose toxicity in NIDDMs. In this regard, it is intriguing to speculate whether this acquired affinity defect reflects a general consequence of high prevailing glucose concentrations or rather that NIDDMs are uniquely susceptible to the toxic effects of glucose to induce this defect (for example an abnormal glucose transporter susceptible to glycosylation). Recent data from our laboratory indicate that in a group of type I, insulindependent diabetics with $\mathrm{GHb}$ levels $>14 \%$, the affinity of skeletal muscle for glucose is normal (31). Therefore, the 
current data suggest (but do not prove) that NIDDMs may have a unique susceptibility to the toxic effects of glucose to cause peripheral insulin resistance through the induction of a tissue affinity defect for glucose uptake. The molecular basis for this observation deserves further exploration.

If the lower affinity of peripheral tissues for glucose uptake ( $K_{\mathrm{m}}$ defect) is what differentiates the kinetics of IMGU in severely hyperglycemic NIDDM from simple obesity, how does this $K_{\mathrm{m}}$ defect evolve? The data herein cannot directly address this question but do suggest a testable hypothesis. Because the clinical state of NIDDM evolves from a state of glucose intolerance (30), it is reasonable to expect that even before the onset of overt fasting hyperglycemia the tissues of these patients are exposed to markedly elevated glucose concentration in the postprandial state. According to this hypothesis, after years of exposure to high glucose, the expression of glucose toxicity in these subjects occurs in a unique fashion (possibly because of a genetic susceptibility) such that a $K_{\mathrm{m}}$ defect for IMGU appears in peripheral tissues leading to decreased rates of IMGU. In addition, a defect in glucose sensing appears at the level of the Beta cell (possibly another $\mathrm{Km}$ defect) which leads to decreased insulin secretion (32). In this setting, hepatic glucose output is allowed to accelerate and raise the fasting serum glucose concentration to a level sufficient to normalize rates of IMGU (33). This is best accomplished when the prevailing glucose level is close to the $K_{\mathrm{m}}$ for IMGU, i.e., where glucose clearance is most efficient. Therefore, in this hypothetical construct a selfreinforcing cycle of postprandial hyperglycemia $\rightarrow$ glucose toxicity $\rightarrow$ increased $K_{\mathrm{m}}$ for IMGU fasting hyperglycemia, is in place where the $K_{\mathrm{m}}$ defect for IMGU in peripheral tissues of these patients provides the set point for the prevailing serum glucose level. This hypothesis remains to be tested but it provides a rational basis for the evolution for the phenotypic expression of the NIDDM lesion.

Finally, we have previously reported that both insulin and glucose modulate skeletal muscle blood flow and that this is an important physiological mechanism for the disposal of glucose $(9,10,34)$. Leg blood flow rates among the obese nondiabetics and NIDDMs (particularly in the severe NIDDM group) tended to be lower than those observed in lean controls. Given the importance of glucose and insulin delivery to skeletal muscle to modulate glucose uptake (34), it follows that decreased blood flow rates to skeletal muscle contributed to the overall decrease in the rates of whole body IMGU in the obese groups. We have previously discussed this issue in more detail (9, 10, 34).

Because intensive insulin therapy in severe NIDDMs failed to increase or normalize rates of leg blood flow, it is likely that the incomplete reversal of the "postreceptor" defect in NIDDM patients after insulin therapy as previously reported (11) is due at least in part to a decrease in insulin and glucose delivery (blood flow) to insulin-sensitive tissues, a "prereceptor" defect. By virtue of its proximal locus, this latter defect has the potential to be rate limiting and give rise to a decrease in insulin responsiveness. This issue warrants further study.

In summary, decreased rates of skeletal muscle IMGU in poorly controlled NIDDMs are secondary to decreases in both capacity and affinity of the skeletal muscle glucose uptake system. This defect in tissue affinity for glucose is related to the degree of glycemic control in these patients and is reversible with intensive insulin therapy, suggesting that it is an acquired defect. In contrast, the magnitude of the reduction in tissue capacity for glucose uptake appears to be largely related to the obesity status and is not modulated by the severity of the diabetic state or insulin therapy.In addition to these tissue defects, decreased skeletal muscle blood flow in obesity and NIDDM contribute to decreased rates of whole body IMGU. In conclusion, in vivo insulin resistance to insulin's action to stimulate glucose uptake can be the result of a decrease in skeletal muscle tissue capacity and/or affinity for glucose uptake and/or a decrease in blood flow to insulin-sensitive tissues.

\section{Acknowledgments}

The authors wish to thank Tim Garvey for critical review of the manuscript and Kate Petrey for her invaluable secretarial assistance.

This work was supported in part by grants from M01 RR-00827 and DK42469, National Institutes of Health (NIH), the American Diabetes Association, the Diabetes Research and Education Foundation, and the Juvenile Diabetes Foundation. Dr. Laakso was a visiting scientist from the University of Kuopio, Finland, and was the recipient of a Fogarty International Fellowship Grant, NIH.

\section{References}

1. DeFronzo, R. A., E. Jacot, E. Jequier, E. Maeder, J. Wahren, and J. P. Felber. 1981. The effect of insulin on the disposal of intravenous glucose. Results from indirect calorimetry and hepatic and femoral venous catheterization. Diabetes. 30:1000-1007.

2. Baron, A. D., G. Brechtel, P. Wallace, and S. V. Edelman. 1988. Rates and tissue sites of non-insulin and insulin-mediated glucose uptake in humans. Am. J. Physiol. 255:E769-E774.

3. Dohm, E. J., E. B. Tapscott, W. J. Pories, D. J. Dabbs, E. G. Flickinger, D. Meelheim, T. Fushiki, S. A. Atkinson, C. W. Elton, and J. F. Caro. 1988. An in vitro human muscle preparation suitable of metabolic studies. Decreased insulin stimulation of glucose transport in muscle from morbidly obese and diabetic subjects. J. Clin. Invest. 82:486-494.

4. DeFronzo, R. A., R. Gunnarsson, O. Bjorkman, M. Olson, and J. Wahren Effects of insulin on peripheral and splanchnic glucose metabolism in non-insulin dependent (type II) diabetes mellitus. J. Clin. Invest. 76:149-155.

5. Kolterman, O. G., R. S. Gray, J. Griffin, P. Burstein, J. Insel, J. A. Scarlett, and J. M. Olefsky. 1981. Receptor and postreceptor defects contribute to the insulin resistance in non-insulin dependent diabetes mellitus. J. Clin. Invest. 68:957-969.

6. Garvey, T. W., T. P. Huecksteadt, S. Matthaei, and J. M. Olefsky. 1988. Role of glucose transporters in the cellular insulin resistance of type II non-insulin-dependent diabetes mellitus. J. Clin. Invest. 81:1528-1536.

7. Ciaraldi, T. P., O. G. Kolterman, J. A. Scarlett, and J. M. Olefsky. 1982. Role of glucose transport in the post-receptor defect of non-insulin dependent diabetes mellitus. Diabetes. 31:1016-1022.

8. Revers, R. R., O. G. Kolterman, and J. M. Olefsky. 1983. Relationship between serum glucose level and the metabolic clearance rate of glucose in noninsulin dependent diabetes mellitus. Diabetes. 32:627-632.

9. Edelman, S. V., M. Laakso, P. Wallace, G. Brechtel, J. M. Olefsky, and A. D. Baron. 1990. Kinetics of insulin mediated and non-insulin-mediated glucose uptake in humans. Diabetes. 39:955-964.

10. Laakso, M., S. V. Edelman, J. M. Olefsky, G. Brechtel, P. Wallace, and A. D. Baron. 1990. Kinetics of in vivo muscle insulin mediated glucose uptake in human obesity. Diabetes. 39:965-974.

11. Garvey, W. T., J. M. Olefsky, J. Griffin, R. F. Hamman, and O. G. Kolterman. 1985. The effect of insulin treatment on insulin secretion and insulin action in type II diabetes mellitus. Diabetes. 34:222-234.

12. Andrews, W. J., B. Vasquez, M. Nagulesparan, L. Klimes, J. Foley, R. Unger, and G. H. Reaven. 1984. Insulin therapy in obese, non-insulin-dependent diabetes induces improvement in insulin action and secretion that are maintained for two weeks after insulin withdrawal. Diabetes. 30:634-642.

13. National Diabetes Data Group. 1979. Classification and diagnosis of diabetes mellitus and other categories of glucose intolerance. Diabetes. 28:10391057.

14. DeFronzo, R. A., J. D. Tobin, and R. Andres. 1979. Glucose clamp technique: a method for quantifying insulin secretion and resistance. Am. J. Physiol. 237:E214-E223.

15. Prager, R., P. Wallace, and J. M. Olefsky. 1986. In vivo kinetics of insulin action on peripheral glucose disposal and hepatic glucose output in normal and obese subjects. J. Clin. Invest. 78:472-481. 
16. Molina, J. M., P. Wallace, T. Flynn, R. R. Henry, and B. Gumbiner. 1989 Weight loss abolishes defect in deactivation but not activation of insulin action in NIDDM. Diabetes. 38(Suppl.):68A.

17. Zierler, K. L. 1961. Theory of the use of arteriovenous concentration differences for measuring metabolism in steady and non-steady state. J. Clin. Invest. 40:2111-2125.

18. Desbuquois, B., and G. D. Aurbach. 1971. Use of polyethylene glycol to separate free and antibody bound peptide hormones in radioimmunoassays. $J$. Clin. Endocrinol. Metab. 33:732-738.

19. DeLean, A. P., P. J. Munson, and D. Rodbard. 1978. Simultaneous analysis of families of sigmoidal curves: application to bioassay, radioligand assay, and physiological dose-response curves. Am. J. Physiol. 235:E97-E102.

20. Bogardus, C., S. Lillioja, B. V. Howard, G. Reaven, and D. Mon 1984 Relationship between insulin secretion, insulin action and fasting plasma glucose concentration in non-diabetic and non-insulin-dependent diabetic subjects. $J$. Clin. Invest. 74:1238-1246.

21. Best, J. D., R. G. Judzewitsch, M. A. Pfeiffer, J. C. Beard, J. B. Halter, and D. Porte, Jr. 1982. The effect of chronic sulfonylurea therapy on hepatic glucose production in non-insulin-dependent diabetes. Diabetes. 31:333-338.

22. Simonson, D. C., E. Ferrannini, S. Bevilacqua, D. Smith, E. Barrett, R Carlson, and R. A. DeFronzo. 1984. Mechanism of improvement in glucose metabolism after chronic glyburide therapy. Diabetes. 33:838-845.

23. Henry, R. R., P. Wallace, and J. M. Olefsky. 1986. Effects of weight loss on mechanisms of hyperglycemia in obese non-insulin-dependent diabetes mellitus. Diabetes. 35:990-998.

24. Zawadzki, J. K., C. Bogardus, and J. E. Foley. 1987. Insulin action in obese non-insulin-dependent diabetics and in their isolated adipocytes before and after weight loss. Diabetes. 36:227-235.
25. Ziel, F. H., N. Venkatesan, and M. B. Davidson. 1988. Glucose transport is rate limiting for skeletal muscle glucose metabolism in normal and STZ-induced diabetic rats. Diabetes. 37:885-890.

26. Katz, A., B. L. Nyomba, and C. Bogardus. 1988. No accumulation of glucose in human skeletal muscle during euglycemia hyperinsulinemia. $\mathrm{Am}$. J Physiol. 255:E942-E945.

27. Karnieli, E., A. Barzilai, R. Rafaeloff, and M. Armoni. 1986. Distribution of glucose transporters in membrane fractions isolated from human adipose cells. Relation to cell size. J. Clin. Invest. 78:1051-1055.

28. DeFronzo, R. A. 1988 . The triumvirate: beta-cell, muscle, liver: a collusion responsible for NIDDM. Diabetes. 37:667-687.

29. Scarlett, J. A., R. S. Gray, J. Griffin, J. M. Olefsky, and O. G. Kolterman. 1982. Insulin treatment reverses the insulin resistance of type II diabetes mellitus. Diabetes Care. 5:353-363.

30. Rosetti, L., D. Smith, G. I. Shulman, D. Papachristou, and R. A. DeFronzo. 1987. Correction of hyperglycemia with phlorizin normalizes tissue sensitivity to insulin in diabetic rats. J. Clin. Invest. 79:1510-1515.

31. Baron, A. D., M. Laakso, G. Brechtel, and S. V. Edelman. 1990. Mechanisms of insulin resistance in insulin dependent diabetics. A major role for reduced skeletal muscle blood flow. Diabetes. 39(Suppl.):342. (Abstr.)

32. Robertson, R. P. 1989. Type II diabetes, glucose "non-sense," and islet desensitization. Diabetes. 38:1501-1505.

33. Baron, A. D., O. G. Kolterman, J. Bell, L. J. Mandarino and J. M. Olefsky. 1985. Rates on non-insulin mediated glucose uptake are elevated in type II diabetic subjects. J. Clin. Invest. 76:1782-1788.

34. Laakso, M., S. V. Edelman, G. Brechtel, and A. D. Baron. 1990. Decreased effect of insulin to stimulate skeletal muscle blood flow in obese man: a novel mechanism for insulin resistance. J. Clin. Invest. 85:1844-1852. 